\title{
Structural Analysis of Molecular Clouds: Dendrograms
}

\section{Citation}

Rosolowsky, E. W., J. E. Pineda, J. Kauffmann, and A. A. Goodman. 2008. "Structural Analysis of Molecular Clouds: Dendrograms." The Astrophysical Journal 679 (2): 1338-51. https:// doi.org/10.1086/587685.

\section{Permanent link}

http://nrs.harvard.edu/urn-3:HUL.InstRepos:41397498

\section{Terms of Use}

This article was downloaded from Harvard University's DASH repository, and is made available under the terms and conditions applicable to Open Access Policy Articles, as set forth at http:// nrs.harvard.edu/urn-3:HUL.InstRepos:dash.current.terms-of-use\#OAP

\section{Share Your Story}

The Harvard community has made this article openly available.

Please share how this access benefits you. Submit a story.

Accessibility 
DRAFT VERSION OCTOBER 28, 2018

Preprint typeset using LTEX style emulateapj v. 08/22/09

\title{
STRUCTURAL ANALYSIS OF MOLECULAR CLOUDS: DENDROGRAMS
}

\author{
E. W. Rosolowsky ${ }^{1,2}$, J. E. Pineda ${ }^{1}$, J. Kauffmann ${ }^{1,3}$, And A. A. Goodman ${ }^{1,3}$ \\ Draft version October 28, 2018
}

\begin{abstract}
We demonstrate the utility of dendrograms at representing the essential features of the hierarchical structure of the isosurfaces for molecular line data cubes. The dendrogram of a data cube is an abstraction of the changing topology of the isosurfaces as a function of contour level. The ability to track hierarchical structure over a range of scales makes this analysis philosophically different from local segmentation algorithms like CLUMPFIND. Points in the dendrogram structure correspond to specific volumes in data cubes defined by their bounding isosurfaces. We further refine the technique by measuring the properties associated with each isosurface in the analysis allowing for a multiscale calculation of molecular gas properties. Using COMPLETE ${ }^{13} \mathrm{CO}(J=1 \rightarrow 0)$ data from the L1448 region in Perseus and mock observations of a simulated data cube, we identify regions that have a significant contribution by self-gravity to their energetics on a range of scales. We find evidence for self-gravitation on all spatial scales in L1448 though not in all regions. In the simulated observations, nearly all of the emission is found in objects that would be self-gravitating if gravity were included in the simulation. We reconstruct the size-line width relationship within the data cube using the dendrogram-derived properties and find it follows the standard relation: $\sigma_{v} \propto R^{0.58}$. Finally, we show that constructing the dendrogram of CO $(J=1 \rightarrow 0)$ emission from the Orion-Monoceros region allows for the identification of giant molecular clouds in a blended molecular line data set using only a physically motivated definition (self-gravitating clouds with masses $\left.>5 \times 10^{4} M_{\odot}\right)$.

Subject headings: ISM:clouds — ISM: structure — methods: analytical — techniques: image processing
\end{abstract}

\section{INTRODUCTION}

The structure in molecular clouds determines, in part, the locations, numbers and masses of newly formed stars. Because of its important role at establishing the initial mass function of stars as well as the local star formation rate, great effort has been invested in characterizing the structure of this gas. Observations of molecular clouds including molecular and atomic line surveys, extinction and infrared emission mapping, and star counts have all been used to characterize the nature of molecular clouds. A myriad of analytic techniques have been applied to these data with a broad range of results. Each technique is designed to highlight a different feature of the gas: fractal analysis techniques are used to demonstrate that the gas is fractal (Stutzki et al. 1998); searches for clumps utilize clump identification algorithms (Stutzki \& Güsten 1990); studies characterizing turbulence frequently aim to measure theoretically relevant quantities such as the power spectrum (Lazarian \& Pogosyan 2000) or the structure function (Heyer \& Brunt 2004).

One of the dominant characteristics of molecular gas it that it is hierarchical. A preponderance of multi-tracer studies have consistently shown that the high-density features in molecular clouds have relatively small physical scales and are invariably contained inside envelopes of lower density gas (e.g. Blitz \& Stark 1986; Lada 1992). Moreover, the hierarchy is non-trivial: for any given scale, there are more smallscale, dense structures than there are large-scale, sparse structures. Dense cores are the top level of the cloud hierarchy and the turbulence that characterizes molecular clouds makes

\footnotetext{
Electronic address: erosolow@cfa.harvard.edu

${ }^{1}$ Center for Astrophysics, 60 Garden St., Cambridge, MA 02138

${ }^{2}$ University of British Columbia Okanagan, 3333 University Way, Kelowna, BC V1V 1V7, Canada

${ }^{3}$ Initiative for Innovative Computing, Harvard University, 60 Oxford St. Cambridge, MA 02138
}

a transition to coherence (i.e. domination by thermal rather than turbulent motions) on $\ell \sim 0.1 \mathrm{pc}$ scales (Goodman et al. 1998; Tafalla et al. 2004; Lada et al. 2007). These dense cores are the exclusive hosts of star formation inside molecular clouds and much effort has been expended to study the properties of these cores and the stars that form inside them (e.g., di Francesco et al. 2007; Ward-Thompson et al. 2007, and references therein). Indeed, studies have argued for the close relationship between the dense cores and the newlyformed stars based on the similarities of their mass functions (Motte et al. 1998; Testi \& Sargent 1998; Alves et al. 2007; Enoch et al. 2006).

The low-density gas that fills the majority of the volume of the molecular cloud can be regarded as the bottom of the gas hierarchy in the molecular clouds (though the filling fraction and chemical state of the molecular cloud is far from uniform). The chemical change associated with the formation of star-forming (molecular) clouds has commonly been used to define discrete clouds in the interstellar medium (ISM) serving as a useful division between the diffuse, multi-phase ISM and star-forming clouds. However, there is some debate about whether the boundaries of molecular clouds form a meaningful bottom of the hierarchy and are distinct entities (the "classical" interpretation, Blitz et al. 2007); or whether the hierarchical structure continues with only chemical changes into the diffuse ISM (e.g. Ballesteros-Paredes et al. 1999; Hartmann et al. 2001). The crux of the debate centers around lifetimes of the molecular clouds relative to their internal crossing times or, equivalently, the importance of self-gravity in the cloud's energetics. However, much of this debate has centered on considering disparate sets of observations and Elmegreen (2007) has presented a synthesis that argues relatively long-lived (20-30 Myr) self-gravitating clouds can accommodate local, rapid star formation within them accounting for the sets of observations that drive an apparent contra- 
diction in measurements of cloud lifetimes.

Connecting molecular clouds to the atomic gas in and around them is particularly difficult since the $21-\mathrm{cm}$ observations that would be directly comparable to molecular line studies suffer from fore- and background confusion as well as an intrinsic degradation of spatial resolution from the long wavelength of the emission. For some cases, where geometry (Pound \& Goodman 1997), self-absorption (Li \& Goldsmith 2003), or modeling of photodissociation regions (Bensch 2006) allows, the atomic gas related to molecular clouds can be studied. Studying the hierarchical structure within molecular clouds requires a large spatial dynamic range which restricts useful observational data sets to galactic objects. Although the hierarchical structure of the ISM continues to large scales in the galaxies, the above restrictions limit considerations of hierarchical structure within star forming clouds to those found in the gas traced by molecular emission.

This paper presents another analytic technique aimed to characterize the hierarchical structure in molecular gas and relate it to the star formation process. We use dendrograms to graphically represent hierarchical structure of nested isosurfaces in three-dimensional molecular line data cubes (i.e. position-position-velocity data cubes). The dendrograms are abstractions of how the isosurfaces nest inside one another. Our principal contribution in this work is using standard molecular line analysis techniques to characterize the branches in a dendrogram allowing for simultaneous measurement of various properties on a range of physical scales. In addition, dendrograms are a reduction of the structure in a data set to its essential features and, as such, they become useful reductions of large data sets to simple models allowing the study of a wide range of spatial scales.

The dendrograms presented here are simply an alternative application of the structure trees presented first in Houlahan \& Scalo (1992, hereafter HS92). While novel at the time for the star formation community, such diagram techniques were relatively common in other disciplines (West 2000). In the intervening time since the publication of HS92, the analysis of tree networks has become even more developed and tools for the construction and analysis of the resulting structure trees have become commonplace (e.g. we will apply software in the standard IDL distribution for the following analysis). Our application of the dendrogram formalism uses a significantly different analytic approach compared to the work of HS92. They analyzed the characteristics of the structure trees derived from two-dimensional data. The present work uses dendrograms as an abstraction of the isosurfaces present in three-dimensional data, emphasizing the properties of those isosurfaces. Finally, note that the application of dendrograms to contour surfaces as in this work and HS92 is significantly different from their common application in statistical analysis (e.g. Ghazzali et al. 1999) where they are used to represent clustering in statistical data sets. We refer to HS92's structure trees as dendrograms to be consistent with the nomenclature adopted in other fields, in particular that of the statistical description of hierarchical systems.

This paper briefly discusses different approaches to molecular line data $(\$ 2)$ before developing the concept of dendrograms (\$3). We discuss several refinements of the dendrogram technique including accounting for the effects of noise (\$3.1), measuring cloud properties on dendrogram branches $(\$ 4)$, and the complications of mapping between observed and physical domains (\$4.1). We conclude with two applications of the dendrogram technique: an analysis of self-gravity in L1448
(\$5) and the identification of GMCs in blended data sets (\$6).

\section{THE ANALYSIS OF MOLECULAR LINE DATA}

Broadly speaking, the statistical analysis of molecular line data has usually followed one of two paths. Either authors construct statistical descriptions of the emission from an entire molecular line data set, or authors will segment (divide) the data into what they believe to be physically relevant structures and study the distribution of properties in the resulting population of objects. Common examples of statistical analysis include fractal analysis (Elmegreen \& Falgarone 1996; Stutzki et al. 1998), $\Delta$-variance (Stutzki et al. 1998; Bensch et al.|2001), correlation functions (Houlahan \& Scalo 1990; Rosolowsky et al. 1999; Lazarian \& Pogosyan 2000) and Principal Component Analysis (Hever \& Brunt 2004). Statistical analyses produce many interesting comparisons between and among data, but the physical interpretation of the statistics can be complicated. The most useful applications of the statistical approach tend to be in comparative measurements between two observational data sets or between observations and a simulation (e.g. Padoan et al. 2006).

The segmentation and identification techniques are favored in the case where the emission is thought to be comprised of physically important substructures. In molecular line astronomy, the classic examples of the segmentation approach is the generation of GMC catalogs for the inner galaxy where GMCs are identified as connected regions of emission above a threshold intensity (Solomon et al. 1987; Scoville et al. 1987). Unfortunately, the results of this approach is controlled by the sensitivity and resolution of the data $\operatorname{set}^{4}$. The two applications of the segmentation approach that have most shaped molecular line astronomy, particularly with regards to the field of star formation, are the clump identification algorithms of Williams et al. (1994) and Stutzki \& Güsten (1990). The clumpy substructure of molecular clouds was first identified by eye (Blitz \& Stark 1986) and this structure is thought to be important at establishing the sites of star formation. Williams et al. (1994) applied a watershed segmentation algorithm to molecular cloud data to identify "clumps" within the cloud (the now-famous CLUMPFIND algorithm). The CLUMPFIND algorithm has spawned many subsequent applications and its utility is discussed elsewhere (Pineda et al. in preparation). Where CLUMPFIND is driven by the structure in the data and precludes finding overlapping objects, the GAUSSCLUMP algorithm of Stutzki \& Güsten (1990) (later revisited by Kramer et al. 1998) iteratively fits threedimensional Gaussians to data cube to identify structures in the data. Both algorithms have been used to define the mass spectrum of clumps within molecular clouds, usually finding $\alpha \sim-1.5$ to -1.9 for $d N / d M \propto N^{\alpha}$. It should be noted that CLUMPFIND and GAUSSCLUMP are not intended to produce the same partitioning of a data cube; they adopt substantially different starting assumptions with a corresponding difference in the results. The results CLUMPFIND and, to a lesser extent, GAUSSCLUMP are influenced by their user-defined parameters and algorithmic design which are designed to mimic the "by eye" identification.

\section{THE DENDROGRAM TECHNIQUE}

The dendrogram technique presented here combines the robustness of the statistical approach with the direct link to

\footnotetext{
${ }^{4}$ Sensitivity and resolution effects also contaminate analysis using statistical methods, but it is possible to correct for these effects (e.g. Bensch et al. 2001).
} 
structure in the data explored in the segmentation and identification approach. The analysis of dendrograms presented in HS92 highlights the utility of the method at characterizing two-dimensional extinction maps using a few simple statistics.

We begin by considering images in general, without a specific astronomical data type in mind. This section emphasizes ideal data where the presence of noise does not interfere with structure identification. As discussed in HS92, a dendrogram is a graphical representation of the primitive structure within an image of arbitrary dimension. It is the skeleton of the object containing only information about the structures and substructures within contour diagram of the object. A schematic of the dendrogram technique is shown in Figure 1 for a onedimensional emission profile. If the emission profile were thresholded $^{5}$ at level $I_{1}$ a single connected region results. In contrast, if the profile is thresholded at $I_{2}$ two distinct objects will result, corresponding to each of the local maxima. The level $I_{\text {crit }}$ represents the critical boundary between these two regimes, below which two objects merge into a single object. The dendrogram is a scheme to track the structure as a function of contour level in the profile and thus it represents the essential information about the structure of the object. The dendrogram also encodes where the composite object combines with the third distinct object.

For two dimensional data, a common analogy is to think of the dendrogram technique as a descriptor of a submerged mountain chain. If the overlying water were drained away, first the peaks of mountains would appear as isolated objects. As successively more water is drained, the peaks would merge together into larger objects. The dendrogram encodes information about which objects merged together and at what contour levels they did so. To plot a dendrogram of this data we can flatten the two dimensional structure into one dimension but doing so eliminates any positional information in the tree.

A useful formalism for interpreting dendrograms in three dimensions is to consider each point in the dendrogram as representing an isosurface ( $3 \mathrm{D}$ contour) in the data cube at a given level. If an arbitrary data set is thresholded at a fixed contour level, it breaks up into one or more distinct regions. The bounding surfaces of these volumes are the isosurfaces represented in the dendrograms, with each distinct surface corresponding to a point in the dendrogram. We identify the distinct surfaces by the set of local maxima that they contain. Over a range of contour intervals with no mergers, thresholding the data at slightly higher or lower level will produce the same essential features, namely the same number of distinct regions containing the same local maxima. Hence, the dendrogram will be comprised of vertical branches. The length of these branches corresponds to the range of contour levels over which a set of isosurfaces is unchanged (though the actual volume will change). There are specific contour levels in the data above which a pair of volumes will be distinct and below which the two volumes are joined. We refer to these critical levels as the merge levels. Below the merge level, a single isosurface contains both sets of local maxima that defined the distinct surfaces above the merge level. To represent this change in the topology of the isosurfaces, we connect the two branches of the dendrogram at the merge level.

A sample dendrogram is shown in Figure 2(top) representing the ${ }^{13} \mathrm{CO}(1 \rightarrow 0)$ emission from the L1448 dark cloud in

\footnotetext{
5 Thresholding is the mapping of a real-valued image to a binary image with all data above the threshold set to 1 and all data below set to 0 .
}

Perseus (Ridge et al. 2006). There is no spatial information encoded in the $x$-axis of the plot but rather the ordering of the leaves is chosen so that the branches of the dendrogram do not cross. This choice facilitates visualizing the hierarchical structure in the data at the expense of retaining the geometrical relations between the leaves. The information on the spatial relationships between the objects is retained in this analysis and can be used to label maps with the regions of dendrograms they correspond to, though it is not shown in the dendrogram. The construction of dendrograms including a discussion of the effects of noise is discussed in more detail below.

\subsection{Determining the Leaves of a Dendrogram}

In the following sections, we specifically consider radioline data cubes in position-position-velocity space (PPV) with intensities given in brightness temperatures (Kelvin). Such observational data are invariably contaminated by noise which interferes with the dendrogram process. The structure of the dendrogram is determined entirely by the local maxima in the data. A local maximum, by definition, has a small region around it containing no data values larger than the local maximum and, hence, a distinct isosurface containing only that local maximum can be drawn. The local maxima determine the top level of the dendrogram, which we refer to as the leaves, defined as the set of isosurfaces that contain a single local maximum.

In noiseless data, every local maximum in the data would correspond to an actual emission feature in the data. Unfortunately, in real data, noise will mask the low-amplitude variations in the emission structure resulting in spurious local maxima that do not correspond to real structure in the data. In the dendrogram method, we suppress the effects of these noise fluctuations by rejecting local maxima that are likely caused by noise.

We describe our algorithm here in more detail considering, without loss of generality, that we are examining only a single cloud of emission such that a low-lying contour will contain all the emission of interest in a cloud. The initial leaves of the dendrogram are selected by identifying all local maxima and then rejecting maxima that are likely to be caused by noise. We generate a list of all local maxima by identifying all pixels in the image that have data values larger than all of their neighbors over a box $D_{\max } \times D_{\max } \times \Delta V_{\max }$ in PPV space where $D_{\max }$ and $\Delta V_{\max }$ are free parameters. A nontrivial box size $\left(D_{\max }\right.$ and $\Delta V$ greater than one pixel/channel) reduces the numbers of candidates that must be checked for significance against our noise-suppression criteria. The algorithm becomes insensitive to structure in the data cube on scales less than a box size. If the box is too large, significant structures are suppressed. Since the rejection of local maxima only simplifies the dendrogram by considering a subset of the structurally defining features, reducing the size of the noisesuppression box can be used to check if an essential feature has been eliminated.

After the initial generation of local maxima, the set is then decimated by removing local maxima that are likely to result from noise. For each pair of candidate maxima, we find the highest shared isosurface that contains both maxima. This isosurface is the merge level, a high-dimensional analog of the contour level at the saddle point shown in Figure 1. For the merge level, we calculate (a) the volume uniquely associated with each maximum and (b) the difference in antenna temperature between the merge level and each local maxi- 

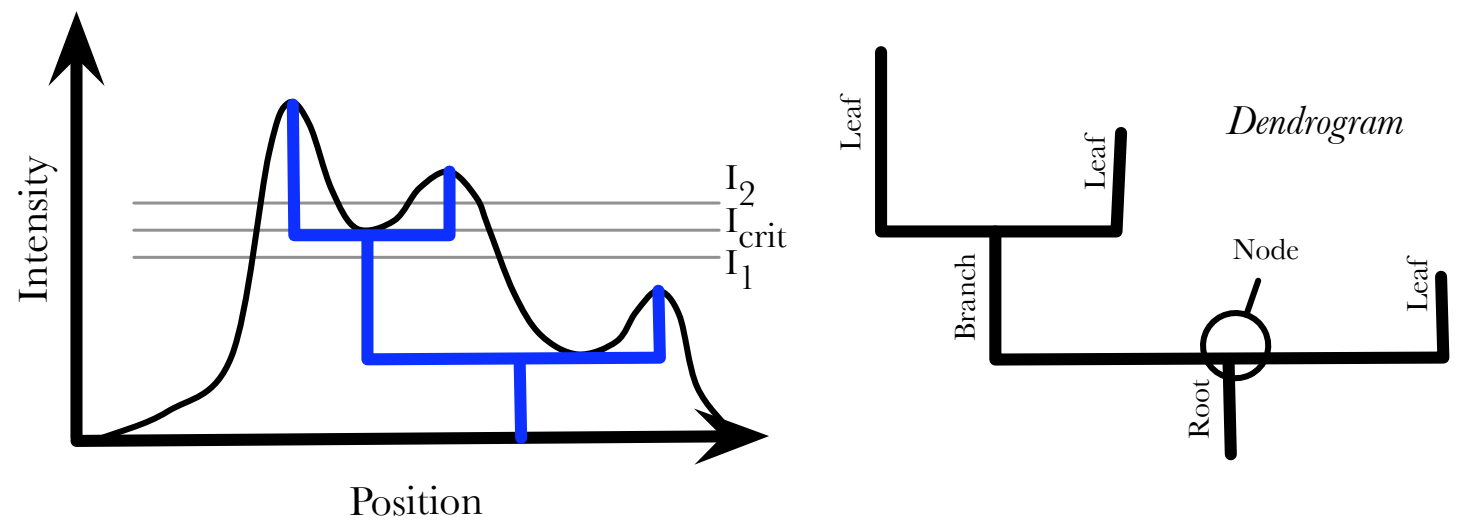

FIG. 1.- Schematic diagram of the dendrogram process. The left panel shows a one-dimensional emission profile with three distinct local maxima. The dendrogram of the region is shown in blue and repeated in the right panel where the components of the dendrogram are labeled. The left-hand panel indicates three characteristic contour levels through the data. Thresholding at $I_{1}$ produces a single object whereas thresholding at $I_{2}$ produces two. The level separating these two regimes is indicated as $I_{\text {crit }}$.

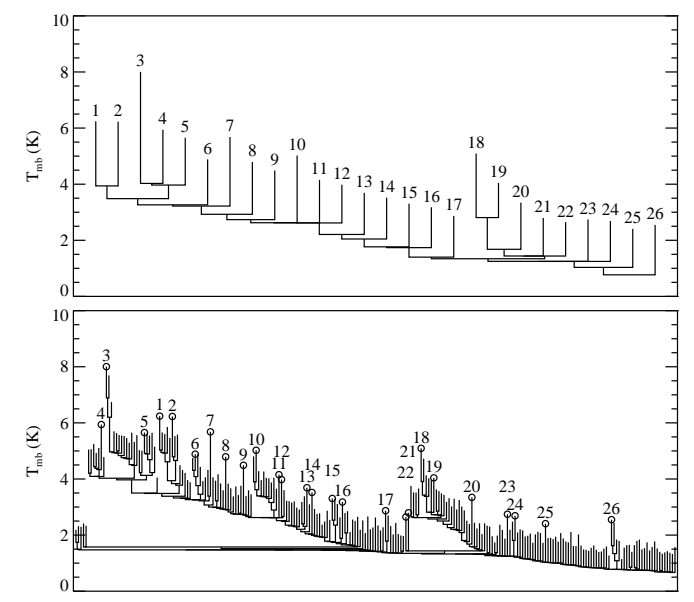

FIG. 2.- Dendrograms of ${ }^{13} \mathrm{CO}$ emission in L1448. The top panel shows the dendrogram of L1448 using the standard algorithm parameters. The bottom panel shows the dendrogram after relaxing the conditions for noise suppression resulting in more independent leaves in the dendrogram (see the end of $\$ 3.1$. However, the basic structure of the dendrogram remains the same; the isosurfaces used in the top plot are a subset of those used in the bottom. Each leaf of the dendrogram is labeled in the top plot and the corresponding leaf is identified in the bottom figure. Leaves appearing in both dendrogram also have a circle at their tip in the bottom plot.

mum. We remove any local maximum for which the volume of the isosurfaces that contain only that maximum is less than some minimum number of pixels ( $N_{\min }$, usually taken to be 4). Furthermore, we only recognize a significant bifurcation in structure when both local maxima are more than a given interval $\Delta T_{\max }$ above the highest contour level that contains both of the maxima, i.e. the level at which the objects merge. Such a criterion has been used previously in data cube analysis (Brunt et al. 2003; Rosolowsky \& Blitz 2005): noise fluctuations will typically only produce maxima with characteristic height $\sigma_{r m s}$ so variations significantly larger than this are nominally real. If this criterion is not fulfilled, we reject the lower of the two local maxima and consider the emission profile to represent only a single object. We note that the resulting dendrogram using a decimated set of local maxima represents a set of isosurfaces that are a subset of the isosurfaces that would be considered including all local maxima (see Figure 2).

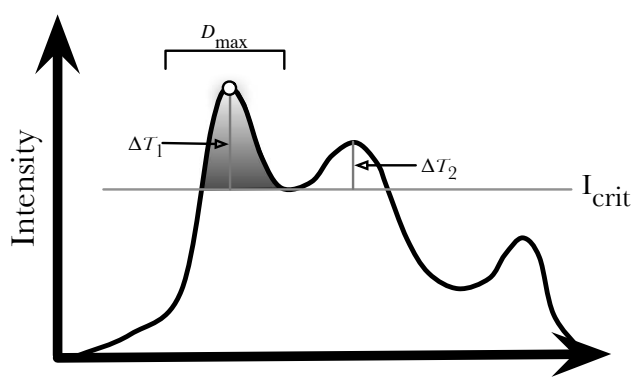

Position

FIG. 3.- Schematic diagram of the parameters that determine the decimation of local maxima. The same profile as in Figure 1 is used. The local maximum indicated with the white point would be considered a valid local maximum if (a) it is the highest point in a window $D_{\max }$ on either side of it (and an analogous width $\Delta V_{\max }$ in velocity space), (b) the interval between the maximum and the highest merger level with a valid local maximum $\Delta T_{1}>\Delta T_{\max }$ and if the number of pixels associated with the shaded region is larger than $N_{\text {min }}$. These criteria restrict the analysis to the subset of local maxima that are most distinct.

Hence, the initial leaves of the dendrogram are determined by four free parameters: $D_{\max }, \Delta V_{\max }, \Delta T_{\max }$ and the $N_{\min }$. By default, these are set to be $D_{\max }=3$ and $\Delta V_{\max }=7$ resolution elements, $\Delta T_{\max }=4 \sigma_{\text {rms }}$ and $N_{\min }=4$ pixels for independent pixels. The bottom panel of Figure 2 shows the resulting dendrogram for $D_{\max }=1$ and $\Delta V_{\max }=3$ resolution elements and $\Delta T_{\max }=0$. Figure 3 is a schematic diagram illustrating the definition of these parameters. Changing $\Delta T_{\max }$ results in the largest changes in the dendrograms for typical radio line data since a larger fraction of the local maxima fail the check against the contrast than any other noise suppression criterion. The default values represent a compromise between sensitivity to dendrogram structure and algorithm performance.

Noise has an additional affect on the dendrogram, namely intensity fluctuations can alter the levels at which two isosurfaces merge. A positive fluctuation can join two surfaces at a higher level than the surfaces would join in the absence of noise. We have modeled the influence of the noise by comparing the merge levels of surfaces in a model cube in the absence of noise to those with noise added. We find, in general, that the merge levels are uncertain on a scale of $\sim 2 \sigma_{\text {rms }}$ with some variation based on algorithm parameters and the 
precise model used. In addition, there is a bias towards merging $\sim 1 \sigma_{r m s}$ higher than the surfaces would merge in the absence of noise. The structure of the tree can only be considered accurate for amplitude changes larger than $\sim 2 \sigma_{r m s}$, and for scales smaller than this, the branching order may be transposed. HS92 discuss this effect in some detail for 2D images and resort to a coarse binning of their trees to measure tree statistics (number of branches per node, etc.). We do not present tree statistics in this paper (see HS92), and we make an effort to account for the influence of noise in our results.

\subsection{Constructing the Dendrogram}

Practically, the dendrogram of an $N$-dimensional intensity image is constructed by first identifying the local maxima that will comprise the top level of the dendrogram hierarchy (\$3.1). Then, the data are contoured with a large number of levels. For each contour value beginning with the maximum level, the dendrogram algorithm checks whether each pair of previously distinct regions have merged together. If so, the contour level and which surfaces merged are recorded and the next contour level is considered. We enforce binary mergers: if three or more distinct objects merge into a single object between one contour level and the next, we refine the separation between contour levels so each merger involves only two objects. The dendrogram (tree diagram) is constructed by drawing vertical segments corresponding to contour levels where the topology of the surfaces are unchanged and connecting corresponding branches at the levels where isosurfaces merge.

Both the identification of local maxima and the levels at which two surfaces can be considered to be merged are influenced by the choice of connectivity in the data set. Practically, astronomical data is pixellated into square (cubic) pixels. The connectivity of the data set is determined by the number of neighbors a given pixel is defined to have. In two dimensions, a pixel can have either four neighbors (those pixels that share edges of the pixel) or eight neighbors (those pixels that share corners or edges). In three dimensions, a cubic pixel can have either six neighbors (those pixels which share a face with the cubic pixel) or 26 neighbors (those pixels which share a face, edge or corner with a given pixel). An alternative definition in the three dimensional case considers a cubic pixel to have 18 neighbors corresponding to those pixels which share a face or an edge, but we emphasize 6- and 26-connectivity in the three dimensional case to be analogous to the two-dimensional case (see Williams et al. 1994, for further discussion). Two points are in the same region if a path can be drawn from one point to the other through connected pixels which are all in the same region. For our analysis, we choose the minimum connectivity (4 neighbors in the 2D case, 6 in the 3D), but we have experimented with the maximum connectivity. Practically, the dendrogram changes by a small degree with corresponding mergers, on average, occurring at higher contour levels since it is "easier" for two regions to connect.

\section{MEASURING CLOUD PROPERTIES IN DENDROGRAMS}

Having developed a formalism where each point in the dendrogram corresponds to a unique isosurface in the data, we calculate the physical properties of the emission bounded by that isosurface. We can then use those physical properties to identify the relevant features in the data cube. Along branches of the dendrogram, the properties tend to be continuous functions of the contour level, while where two branches merge, the properties will change suddenly as a result of the merged object containing more emission. However, owing to the difficulty in relating volumes in observed data space to volumes in physical space, the measurement of properties from regions of emission within the data cube is difficult to interpret (see $\$ 4.1$. In this section, we describe our methods for estimating the size, line width, luminosity and mass contained within each isosurface describe in the dendrogram as well as the complications that arise in doing so.

We calculate the macroscopic properties of the regions of emission based on the moments of the volume weighted by the intensity of emission coming from every pixel following Rosolowsky \& Leroy (2006). The data cube consists of a number of pixels that have sizes of $\delta x, \delta y$, and $\delta v$ in the two spatial dimensions and the velocity dimension, respectively. The $i$ th pixel in the data cube has positions $x_{i}$ and $y_{i}$, velocity $v_{i}$, and brightness temperature $T_{i}$. We assume that the region under consideration is contiguous and bordered by an isosurface in brightness temperature of value $T_{e d g e}$, so that all of the pixels in the region have $T>T_{\text {edge }}$ and the pixels outside the region have $T<T_{\text {edge }}$ or are separated from the region by emission with $T<T_{\text {edge }}$.

We begin by rotating the spatial axes so that the $x$ and $y$ axes align with the major and minor axis of the region, respectively. We determine the orientation of the major axis using principal component analysis. The size of the region is computed as the geometric mean of the second spatial moments along the major and minor axis. This is $\sigma_{r}$, the root-mean-squared (RMS) spatial size:

$$
\sigma_{r}\left(T_{\text {edge }}\right)=\sqrt{\sigma_{\text {maj }}\left(T_{\text {edge }}\right) \sigma_{\min }\left(T_{\text {edge }}\right)}
$$

where $\sigma_{m a j}\left(T_{\text {edge }}\right)$ and $\sigma_{\text {min }}\left(T_{\text {edge }}\right)$ are the RMS sizes derived from the intensity-weighted second moments along the two spatial dimensions.

$$
\sigma_{m a j}^{2}=\frac{\sum_{i} w_{i}\left(x_{i}-\langle x\rangle\right)^{2}}{\sum_{i} w_{i}},
$$

where we have assumed the major axis lies along the $x$ coordinate and the sum runs over all pixels within the isosurface $\left(T>T_{\text {edge }}\right)$. The weights in the moment are usually set to the brightness temperature of each pixel: $w_{i}=T_{i}$. This particular functional form for the cloud size is used since it has been used in previous observational studies (Solomon et al. 1987) and explored in depth by Bertoldi \& McKee (1992) with respect to inclination, aspect ratio, and virialization. We define a factor $\eta$ that relates the one-dimensional RMS size, $\sigma_{r}$, to the radius of a spherical cloud $R: R=\eta \sigma_{r}$. We take $\eta=1.91$ for consistency with Solomon et al. (1987) and Rosolowsky \& Leroy (2006); the value of 1.91 merely reflects the correction of the moment to the radius for the typical concentration of emission found in molecular clouds. The velocity dispersion $\left(\sigma_{v}\right)$ is calculated as the second moment of the velocity axis weighted by the data values, analogous to the size measurement. The flux of the region is the sum (zeroth moment) of all the emission in the region: $F=\sum_{i} T_{i} \delta \theta_{x} \delta \theta_{y} \delta v$. To convert the flux to a luminosity, we must assume a distance to the region. For a cloud at a distance of $d$ (in parsecs), the physical radius will be: $R_{\mathrm{pc}}=R_{\mathrm{rad}} d$ and the luminosity will be $L=F d^{2}$ where the flux is measured in units of $\mathrm{K} \mathrm{km} \mathrm{s}^{-1}$ sr. For $\mathrm{CO}$ data, we calculate the mass of the region, we scale by a linear $\mathrm{CO}-$ to- $\mathrm{H}_{2}$ conversion factor 
(for intensities on the main beam temperature scale):

$$
\frac{M_{\mathrm{Lum}}}{M_{\odot}}=\frac{X_{\mathrm{CO}}}{2 \times 10^{20}\left[\mathrm{~cm}^{-2} /\left(\mathrm{K} \mathrm{km} \mathrm{s}^{-1}\right)\right]} \times 4.4 \frac{L_{\mathrm{CO}}}{\mathrm{K} \mathrm{km} \mathrm{s}^{-1} \mathrm{pc}^{2}} \equiv 4.4 X_{2} L_{\mathrm{CO}} \text {, }
$$

pixels. We can attempt to correct for either of these effects, but not both.

where $X_{\mathrm{CO}}$ is the assumed CO-to- $\mathrm{H}_{2}$ conversion factor. This calculation includes a factor of 1.36 (by mass) to account for the presence of helium. Including helium is necessary to facilitate comparison with the virial mass, which should reflect all of the gravitating mass in the cloud. We have adopted a fiducial value of the CO-to- $\mathrm{H}_{2}$ conversion factor of $X_{\mathrm{CO}}=$ $2 \times 10^{20} \mathrm{~cm}^{-2}\left(\mathrm{~K} \mathrm{~km} \mathrm{~s}^{-1}\right)^{-1}$ based on ${ }^{12} \mathrm{CO}(1 \rightarrow 0)$ observations in the Milky Way (Strong \& Mattox 1996; Dame et al. 2001) and express changes relative to this value in terms of the parameter $X_{2}$.

For each property, we can estimate the uncertainty in the property caused by the noise in the data set. Assuming the coordinate axes are well-defined, the uncertainties are algebraically propagated through the formulas for the physical properties.

\subsection{Physical Interpretation}

The major difficulty in using the above calculated properties directly is that it is difficult to ascribe meaning to a region of emission defined by an isosurface. There is substantial concern that the naive association of a closed object in PPV space with an object in physical space, particularly as defined by contours of intensity in a data cube, may be inaccurate (Ostriker et al. 2001). From the observer's perspective there seem to be three possible interpretations for the emission in the data cube. Each of these interpretations leads to different sets of cloud properties and yields different results when applied to the same data set. These interpretations all revolve around determining what the appropriate values of the antenna temperature weights used in moments of the emission $\left(w_{i}\right)$ should be (e.g., those in Equation 2). We graphically summarize the three "paradigms" for measuring the properties of isosurfaces in Figure 4

\subsubsection{The Bijection Paradigm}

The calculations of properties sets the weights to the native values of brightness temperature drawn from the data cube $w_{i}=T_{i}$. This assumption essentially maps PPV space to physical space (i.e. three spatial dimensions) in a 1-to-1 fashion (one pixel in the data cube corresponds to single volume in the cloud). To the extent that this is true, this is the correct thing to do. This result is the closest parallel to the CLUMPFIND algorithm which associates clumps of emission with clumps of density in physical space. Under the assumption of uniform excitation conditions, an isosurface of brightness corresponds to a surface of constant opacity and hence of constant column density. In the physical regime where higher column densities are associated with higher physical densities, the bijection paradigm may be ideally suited for measuring cloud properties.

A bijection may be inappropriate because of two effects: the first is the superposition of multiple, distinct objects along the line of sight that have the same velocity. The second is that a given volume likely contributes emission at multiple velocities due to an intrinsically broad line profile. Both of these effects cause the bijection to be flawed: the first means that a given pixel contains emission from multiple objects and the second means that any given volume appears in multiple

\subsubsection{The Clipping Paradigm}

In this approach, the region is considered to represent a discrete object superimposed on a background of brightness $T_{\text {edge }}$. This approach assumes that any emission that can be associated with other objects, by drawing a lower contour, is not associated with the object at all. In this case, the properties of the clouds should be calculated using weights $w_{i}=T_{i}-T_{\text {edge }}$. The resulting values of some representative properties are shown in Figure 5 for the set of isosurfaces containing the maximum in the L1448 data cube. In general, the clipping tends to reduce all the properties, but affects the luminosity most significantly. This assumption is very conservative and the correct value would be derived using a weight value intermediate between 0 and $T_{e d g e}$.

\subsubsection{The Extrapolation Paradigm}

In this paradigm, the properties of the region are extrapolated to the zero-intensity isosurface. The extrapolation corrects for the fact that some of the emission arising from the object is not contained within the contour drawn in PPV space. Instead of quoting the properties of the measured region, the extrapolation reports the properties implied for the entire region as inferred from the part found above $T_{\text {edge. }}$. An analogy for this correction is that we are predicting the underwater shape of an island volcano from the visible region above the water.

The extrapolation is carried out by considering the behavior of a property, say $R$, as a function of $T_{\text {edge }}$. For a given $T_{\text {edge }}$, we extrapolate $R\left(T_{\text {edge }}\right)$ to a value of $T_{\text {edge }}=0 \mathrm{~K}$ based on the behavior of $R\left(T_{\text {edge }}^{\prime}\right)$ for all $T_{\text {edge }}^{\prime}>T_{\text {edge }}$. This method is described in more detail in Rosolowsky \& Leroy (2006), in particular in their Figure 2. In short, the second moments are linear extrapolations for data above $T_{\text {edge }}$ whereas the zeroth moments are quadratic extrapolations. The behavior of the extrapolation can be traced on Figure 5. The value of the extrapolated radius is always larger than for the other two paradigms and the margin of difference is most substantial at large contour values since the range of the extrapolation is the largest. At small values of $T_{\text {edge }}$, the extrapolated value oscillates around $\sim 1.5 \mathrm{pc}$, the final radius of the cloud.

It is the latter quadratic extrapolation that produces the noise on the black curve in the left panel of Figure 5. While this method corrects for the emission associated with the object at low intensity values, it effectively adds emission to the objects so that the sum of the extrapolated objects from high intensity values may be larger than the amount of emission contained in the data cube. Hence, ratios such as the virial parameter $(\$ 4.2)$ will be more accurate in this assumption than will integrated properties like radius or mass as a function of contour level.

For the extrapolation paradigm, the dominant source of errors can be the actual data used in the fit and the errors can be assessed by bootstrapping the data used in the extrapolation (Press et al. 1992). See Rosolowsky \& Leroy (2006) for more details.

\subsection{The Virial Parameter}

We adopt the virial parameter as defined in McKee \& Zweibel (1992) as a diagnostic of the energetic state of the regions in the dendrogram. In this case, the 

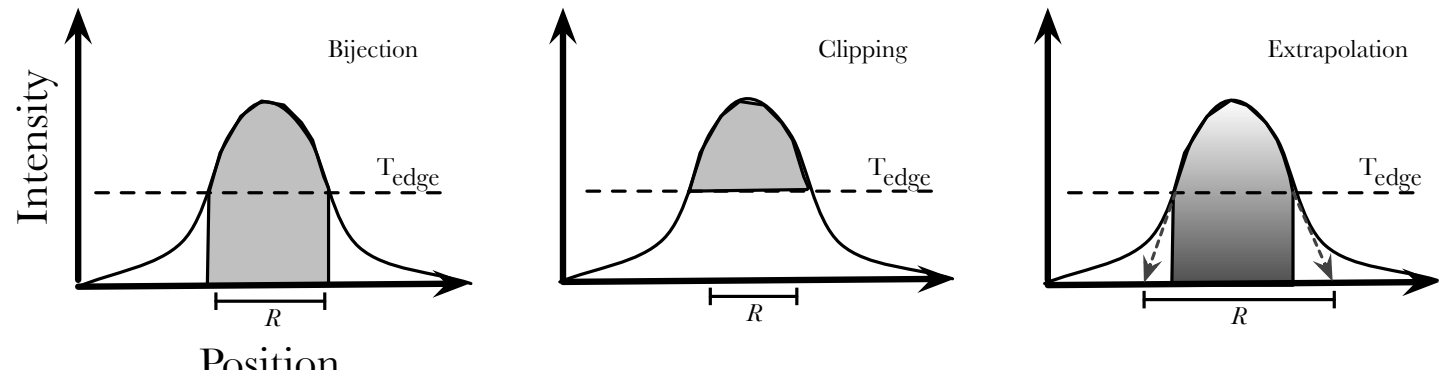

Position

FIG. 4.- Graphical summary of the three paradigms for interpreting isosurfaces of emission investigated in this work. The figure shows the same onedimensional emission profile and a contour level for each of the three cases. The shaded area shows the emission used to compute cloud properties (and is proportional to the luminosity). A bar is shown below the position axis indicating the relative extent or a moment-based size measurement under the three schemes. The standard interpretation of isosurfaces is the bijection scheme where elements in observational space correspond directly to objects in physical space. In the clipping paradigm, only emission above the contour level is associated with an object. In the extrapolation scheme, all the elements above the contour level are used to infer the behavior of the calculated properties in an extrapolation to the zero intensity isosurface. A similar set of characterizations would hold if velocity were the coordinate axis and the line width was measured.
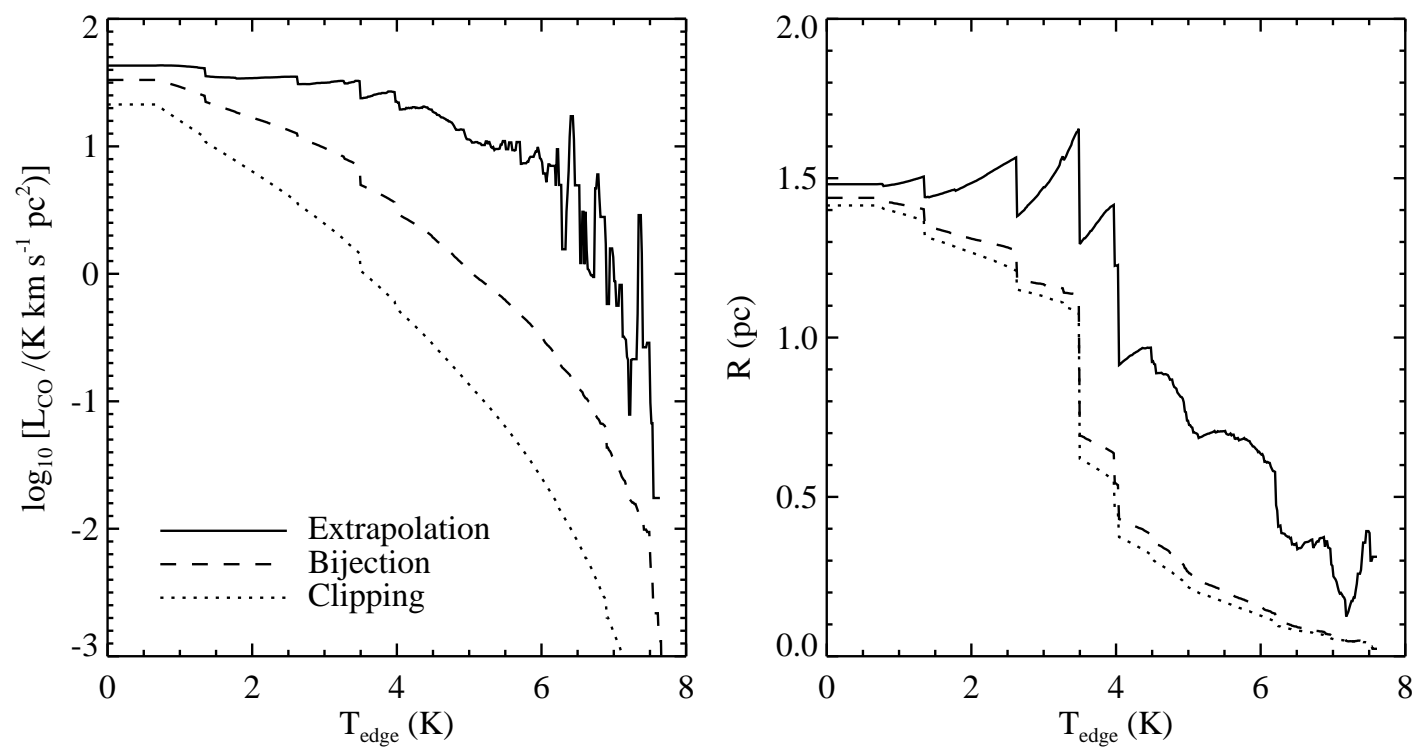

FIG. 5.- The behavior of calculated properties as a function of threshold level in the dendrogram for the set of isosurfaces containing the maximum of the L1448 data cube. The behavior of the cloud properties along the highlighted path are shown for the luminosity (left) and radius (right). Three curves are shown in each of these panels representing three possible ways of calculating the cloud properties at a given $T_{\text {edge }}$. The bijection paradigm is shown as a dashed curve $\left(w_{i}=T_{i}\right)$. The dotted curve depicts the clipping treatment $\left(w_{i}=T_{i}-T_{\text {edge }}\right)$, and the solid curve shows the result of extrapolating the emission to the zero intensity isosurface.

virial parameter $\alpha$ is defined as:

$$
\alpha=\frac{5 \sigma_{v}^{2} R}{M_{\mathrm{Lum}} G}=\frac{5 \eta \sigma_{v}^{2} \sigma_{r}}{4.4 X_{2} L_{\mathrm{CO}} G},
$$

where $L_{\mathrm{CO}}$ is measured in units of $\mathrm{K} \mathrm{km} \mathrm{s}^{-1} \mathrm{pc}^{2}$. For $\alpha<2$, the object is self-gravitating in the absence of other forces. Magnetic fields, surface pressures and bulk motions will all affect the dynamical state of the cloud. Since such terms are not readily measurable, we must adopt this simple estimate for the dynamical state with the understanding that it is only an approximation. The utility of the diagnostic is most likely in a relative sense rather than an absolute one. We should regard regions with $\alpha \lesssim 2$ as regions where significant amounts of gravitational potential (mass) are found with comparably little kinetic energy so that gravity is likely important. In the remainder of the paper, we refer to such regions as "selfgravitating" though the description is subject to the caveats above.

One concern that arises is the meaning of the virial parameter under the three different approaches to calculating the region properties that go into the virial parameter. When adopting the bijection approach, measuring the virial parameter for emission contained above a given contour could be inaccurate since the omission of the "wings" of the cloud would affect the size and line width measurements more than the luminosity measurement. Under the clipping approach, the assumption that none of the emission below a given contour level is associated with the object results in similar size and line widths while dramatically reducing the luminosity. As a result, this assumption likely overestimates the virial parameter for the region. Finally, the virial parameter measured in the extrapolated case characterizes the dynamical state of the region implied by the emission found above a given contour level. The extrapolation method is most useful for character- 
izing objects for which the zero intensity isosurface is a meaningful boundary (i.e. discrete clouds rather than substructure within clouds). Given these considerations and our emphasis on using virial parameter to estimate the dynamical state of structures in the data we adopt the bijection scheme for characterizing substructure, basically interpreting the isosurfaces in the data as corresponding to nested regions of successively higher (column) density. When identifying clouds or other objects for which the zero intensity isosurface is a more meaningful boundary, we adopt the extrapolation paradigm. We discuss the influence of these choices further in $\$ 5.6$ in the application to observed data.

\section{THE HIERARCHICAL SUBSTRUCTURE OF A MOLECULAR CLOUD}

In this section, we apply the dendrogram method to two data sets and demonstrate useful statistics for the characterization of the trees and the tree-based properties.

\subsection{L1448 COMPLETE Data}

Our primary observational data set for demonstrating the dendrogram method is a section of the Coordinated Molecular Probe Line Extinction Thermal Emission ${ }^{6}$ survey's ${ }^{13} \mathrm{CO}$ map of Perseus (Ridge et al. 2006), centered on the L1448 starforming region. The data cube spans a square region $40^{\prime}$ on a side which projects to a region $3.1 \mathrm{pc} \times 3.1 \mathrm{pc}$ at the distance of Perseus (260 pc, Cernis 1993). The data have an angular resolution of $46^{\prime \prime}$ and are sampled with $23^{\prime \prime}$ pixels. Since the observational methods produce non-uniform noise across the map, we add appropriately correlated noise to the original data to produce a map with spatially-uniform noise rms of $\left(\sigma_{r m s}=0.3 \mathrm{~K}\right.$ on the main beam scale). The data cube has a velocity resolution of $0.066 \mathrm{~km} \mathrm{~s}^{-1}$, sampled every $0.066 \mathrm{~km} \mathrm{~s}^{-1}$, and spans $40 \mathrm{~km} \mathrm{~s}^{-1}$, but the emission from L1448 only spans a $10 \mathrm{~km} \mathrm{~s}^{-1}$ section of the data. An integrated intensity map of the cloud is shown in Figure 6 and channel maps are presented in Figure 7. The channel maps highlight the presence of a low velocity feature not otherwise discernible in the integrated intensity maps $\left(v_{\mathrm{LSR}} \sim 0.5 \mathrm{~km} \mathrm{~s}^{-1}\right)$. The main and low velocity features are contained within a single connected isosurface for contour levels $<1.5 \mathrm{~K}$. Individual clumps corresponding to the branches of the dendrograms (see below) can be seen as well as the rough positions of the local maxima used in our analysis of the region (\$5.4).

\subsection{Turbulent Simulation}

For comparison to the data, we also analyze simulation data from Padoan et al. (2006). The data are taken from their simulated ${ }^{13} \mathrm{CO}$ emission maps generated from a 6 pc simulation box with a mean density of $n=10^{3} \mathrm{~cm}^{-3}$. The simulation is conducted using the Enzo code (Norman \& Bryan 1999) to simulate a $1024^{3}$ box using MHD with an initially uniform density and periodic boundary conditions. The mean Mach number in the simulation is $\mathcal{M}=6$; the simulation is isothermal and turbulence is driven in Fourier space at large scales.

For comparison with observed data, Padoan et al. (2006) generated a simulated ${ }^{13} \mathrm{CO}$ data set using a Monte Carlo radiative transfer code using the density and velocity distributions of the simulated material in a snapshot (i.e. radiation is not included in the time propagation of the simulation). We extracted a trial data set matching the spatial extent of the

\footnotetext{
${ }^{6}$ COMPLETE; http: //www. cfa.harvard.edu/COMPLETE/
}

observations from the full simulation box. Our selection included the section of the box that contained the most compact identifiable feature of emission. The trial data were convolved to the resolution of the FCRAO maps from COMPLETE and resampled in position and velocity to match the pixel size of the observational data. Spatially correlated noise, mimicking the noise in the FCRAO map, was added to the simulation data to produce the same underlying noise rms in both data cubes. Both the simulation and the observed data cubes will affected in the same manner by edges, resolution and noise. The only differences between the two data sets should be found in the detailed structure of the emission. The simulation was compared favorably to the full COMPLETE observational data set in Padoan et al. (2006) based on similarities in the turbulent power spectrum. However, the authors of that study emphasize that the simulation is not intended to simulate specific conditions within a molecular cloud. We focused our comparative analysis with this simulation to illustrate the utility of dendrograms even though the simulation box may not be an excellent simulacrum of the L1448 region in particular.

\subsection{The ${ }^{13} \mathrm{CO}$-to- $\mathrm{H}_{2}$ Conversion Factor}

We determine the scaling between ${ }^{13} \mathrm{CO}$ luminosity and molecular cloud mass by comparing the integrated intensity of the ${ }^{13} \mathrm{CO}$ emission to the extinction implied by the reddening of background stars. We use the extinction map for the L1448 region derived from deep JHK Calar Alto observations of the L1448 region using the Near Infrared Color Excess Revisited (NICER Lombardi \& Alves 2001) technique (Foster \& Goodman 2006). The ${ }^{13} \mathrm{CO}$ integrated intensity map is convolved and regridded to match the resolution $\left(48^{\prime \prime}\right)$ and astrometry of the extinction map. The extinction map saturates above $A_{V} \sim 22 \mathrm{mag}$, and we ignore the 13 pixels with missing data in the analysis. Figure 8 shows the implied column density (assuming $N\left(\mathrm{H}_{2}\right) / A_{V}=9.4 \times 10^{20} \mathrm{~cm}^{-2}$ and $R_{V}=3.1$, Bohlin et al. 1978) as a function of the integrated intensity. We calculate a ${ }^{13} \mathrm{CO}-$ to- $\mathrm{H}_{2}$ conversion factor of

$$
X_{13 \mathrm{CO}}=8.0 \times 10^{20}, \frac{\mathrm{cm}^{-2}}{\mathrm{~K} \mathrm{~km} \mathrm{~s}^{-1}}
$$

based on the mean of $N\left(\mathrm{H}_{2}\right) / W\left({ }^{13} \mathrm{CO}\right)$ weighted by the inverse variance of the column density estimates. As seen in Figure 8, the single conversion factor is nowhere an excellent approximation of the data, but represents an adequate mapping between $\mathrm{CO}$ emission and column density over the entire range. Since the conversion factor will be ultimately applied to individual channels, only a simple ratio is appropriate for the relationship; including complications such as nonlinearities or a constant offset would bring up ambiguities in translating from ${ }^{13} \mathrm{CO}$ emission to mass for individual channels in the data cube. The simple ratio systematically underestimates the column density for high brightness regions where the ${ }^{13} \mathrm{CO}$ line saturates. As such, estimates for the virial parameter are likely overestimates in these regions. In terms of the mass calculations presented in $\$ 4, X_{2}=4.0$. This is comparable to the results of more sophisticated analyses of the conversion factor $\left(X_{2}=2.1\right.$; Pineda et al. 2008) though their complex analysis is only applicable for total line intensity.

\subsection{The Dynamical State of L1448}

We generate a dendrogram of the L1448 region with the ${ }^{13} \mathrm{CO}$ data using the methods discussed in $\$ 3$ We identify local maxima over a box that is 2 beam widths on a side and 5 

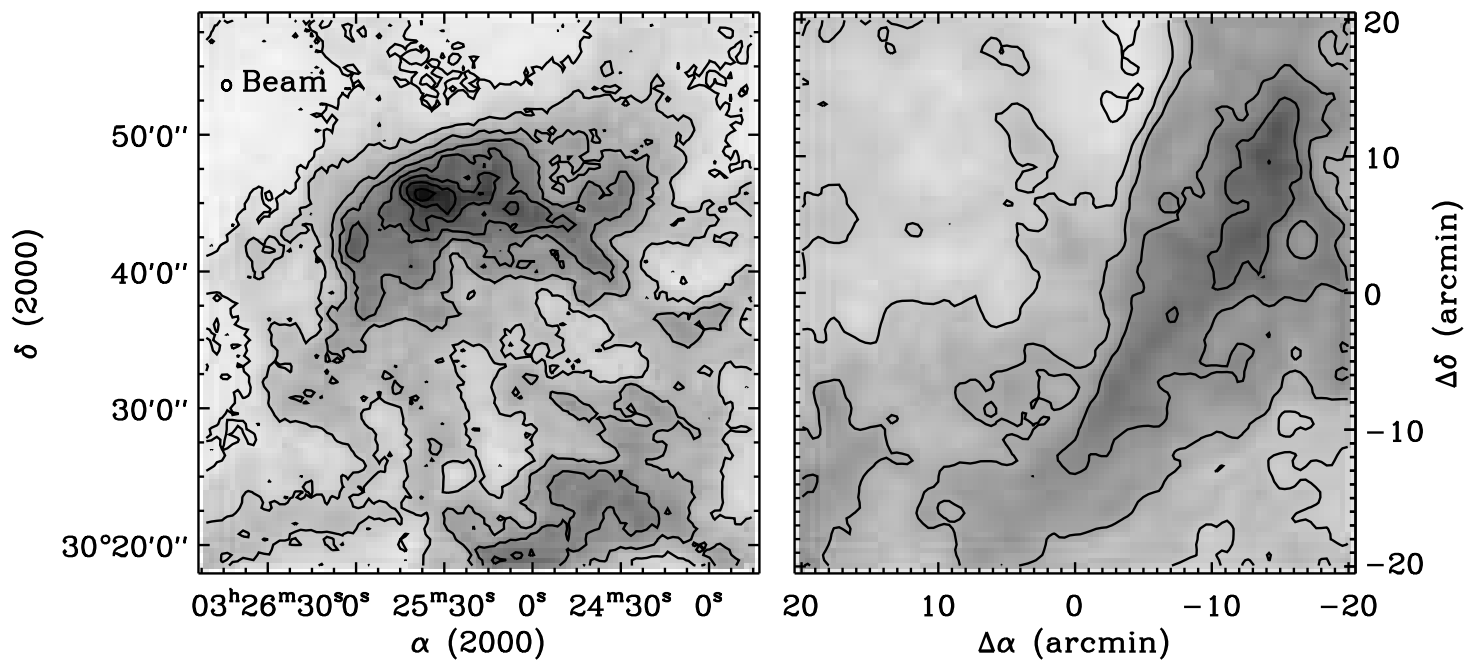

FIG. 6. - Integrated intensity maps of ${ }^{13} \mathrm{CO}$ emission from L1448 (left) and the simulation of Padoan et al. (2006) (right). In both images, the gray scale runs linearly from 0 to $18 \mathrm{~K} \mathrm{~km} \mathrm{~s}^{-1}$ on the $T_{\mathrm{mb}}$ scale and the contours run from $2-16 \mathrm{~K} \mathrm{~km} \mathrm{~s}^{-1}$ in intervals of $2 \mathrm{~K} \mathrm{~km} \mathrm{~s}^{-1}$ in the left-hand panel and from 4-10 K km s in the right-hand panel. The two maps come from data cubes with the same native resolution and noise levels.

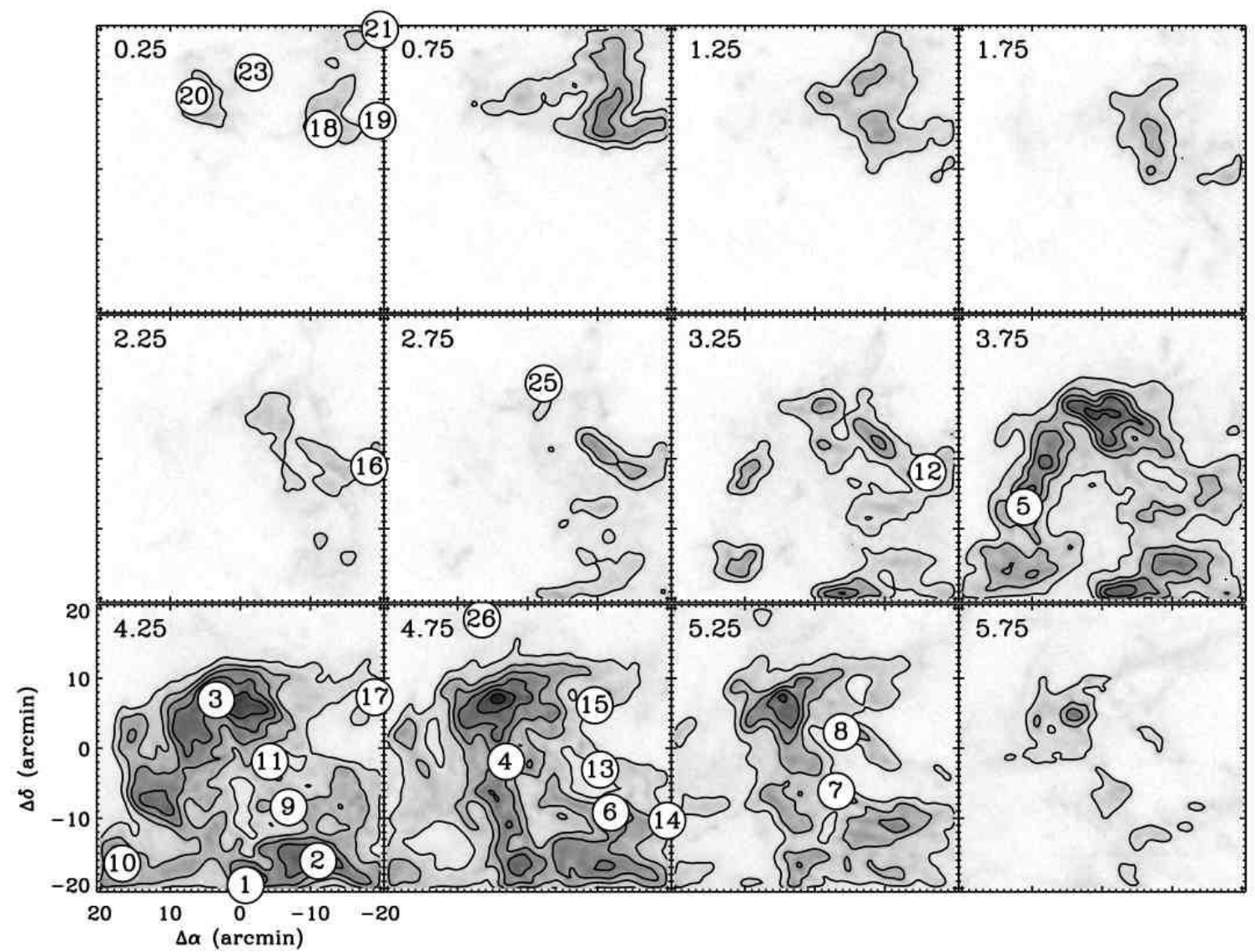

FIG. 7.- Selected channel maps of the L1448 region. Contours follow the grayscale image with a contour interval of $1 \mathrm{~K}$ on the $T_{\mathrm{mb}}$ scale beginning at $1 \mathrm{~K}$. The LSR velocity of each map is indicated in the upper left-hand corner of each panel. The channel maps reveal the low velocity feature $\left(\nu_{\mathrm{LSR}} \sim 0.5 \mathrm{~km} \mathrm{~s} \mathrm{~s}^{-1}\right)$ not otherwise discernible in the integrated intensity map. The 3D positions of the leaves of the dendrogram are indicated with the leaf number shown in Figure 2 shown in the closest channel map to their actual position. 


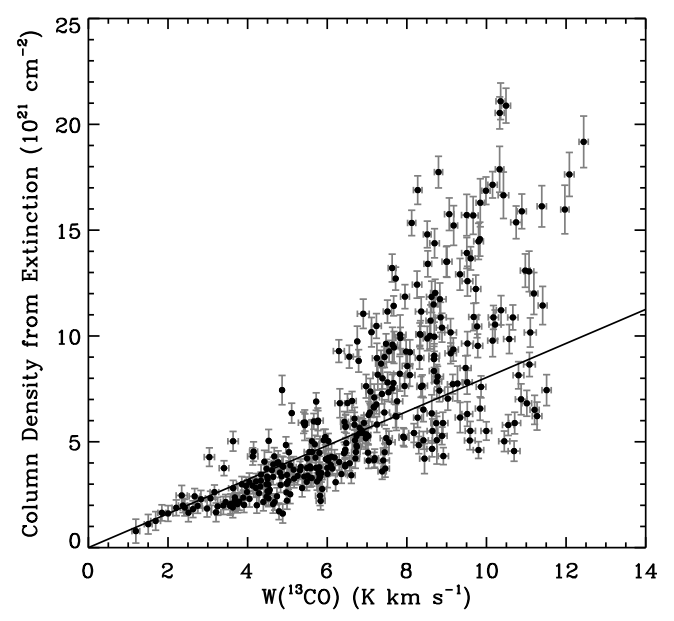

FIG. 8.- Relationship between integrated intensity for ${ }^{13} \mathrm{CO}$ and the $\mathrm{H}_{2}$ column density implied by reddening in the near infrared. A line representing the mean ratio between the two quantities is shown.

channels deep in the data cube. From this set, we eliminate redundant local maxima that are less than $1.2 \mathrm{~K}\left(4 \sigma_{r m s}\right)$ above the level at which the surfaces containing that local maximum merge with other structures. We remind the reader that this decimation preserves the overall structure of the dendrogram and is considering a representative subset of the topologically important surfaces in the analysis (Figure 2). We calculate the virial parameter for the region as discussed in $\$ 4.2$ using the bijection scheme to calculate isosurface properties. In Figure 9 we plot the dendrogram of the region, color coding points on the dendrogram with the corresponding value of the virial parameter. We have calculated the errors in the virial parameter and suppress reporting any values where the formal errors in the virial parameter are larger than 50\%. Figure 9 shows that several leaves of the dendrogram show evidence for self-gravitation on small scales associated with individual local maxima. Note that the left-hand branches (leaves 1-17) of the dendrogram appear self-gravitating, but for contour levels $\lesssim 1.5 \mathrm{~K}$ where the left and right branches merge, the ensemble properties of the object revert to being unbound. This change in dynamical state shows the main complex of L1448 is dynamically distinct from the low velocity feature at $v_{\mathrm{LSR}} \sim 0.5 \mathrm{~km} \mathrm{~s}^{-1}$ indicated by the branch on the right (leaves 18-22).

In general, the tops of the dendrogram leaves do not appear as self-gravitating objects in this analysis. However, it is precisely in these regions where the ${ }^{13} \mathrm{CO}$ tracer saturates so that these isosurfaces correspond to relatively more mass per unit brightness than our simple conversion factor admits and hence will be more tightly bound than we measure. Owing to the difficulties in assessing the dynamical state of these small objects, we emphasize the larger scales for self-gravitation in our analysis. These large scales are where CLUMPFIND and GAUSSCLUMP fail to probe and dendrograms provide novel insight.

The multiscale analysis of the virial parameter allows us to define objects that are potentially physically relevant to the star formation process. We identify objects based on the criterion that self-gravity makes a significant contribution to their internal energetics. If we define a threshold for significant self-gravity, namely $\alpha \leq 2$, we find "interesting" objects on a variety of scales. Applying this criterion to the virial parame-

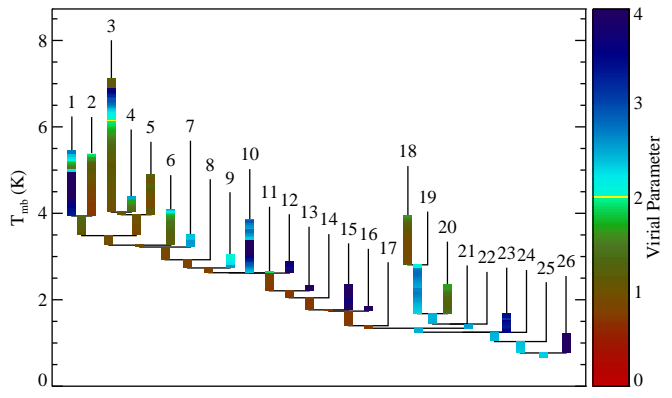

FIG. 9.- Dendrogram of the L1448 region with branches of the dendrogram colored according to the virial parameter at each point. Virial parameter data are suppressed where the errors are larger than $50 \%$. Several of the leaves of the dendrogram show evidence for self-gravitation as do larger structures in data. Since physical properties are calculated for the isosurfaces corresponding to the vertical branches of the figure, the horizontal branches of the dendrogram have no data reported.

ter data shown in Figure 9 results in the dendrogram shown in Figure 11 (left panel) where branches with $\alpha \leq 2$ are shaded. Nearly all of the left-hand branch of the dendrogram corresponds to a self-gravitating object indicating the importance of self-gravity over the entire L1448 region. There are also three distinct sub-branches inside L1448 that also show selfgravitation. Figure 10 shows the locations and spatial extent of the four leaves that show evidence of self-gravitation in the data cube $(2,3,5 \& 18)$. The central, star-forming section of L1448 is contained in leaf 3 . Also interesting are the several branches for which there are large regions with reliable measures of the virial parameter which are not self-gravitating. Referring to Figure 9, these branches have $\alpha \gg 2$. Because of the minimal influence of self-gravity on these structures, we contend that these branches correspond to transient or pressure-confined structures in the physical data.

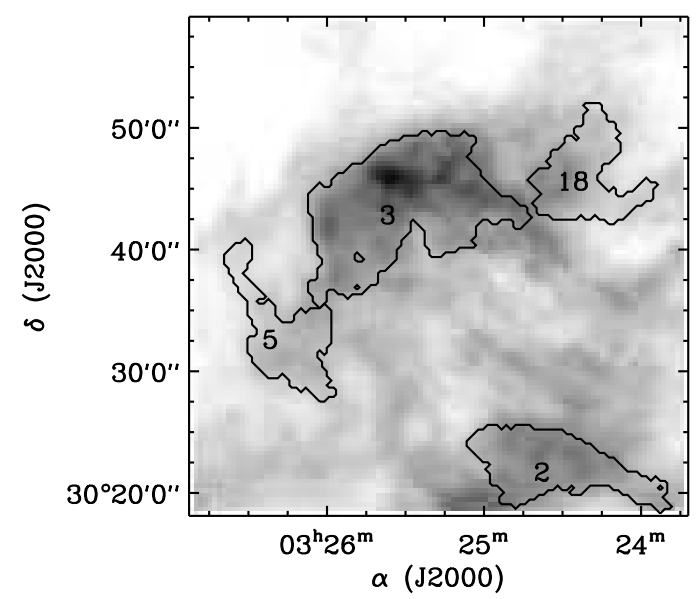

FIG. 10.- Integrated intensity image of the L1448 region with the location and extent of the self-gravitating leaves in the dendrogram indicated. Leaf 3 contains most of the star-formation currently occurring in the region. Leaf 18 is the dynamically distinct feature at low $v_{\mathrm{LSR}}\left(\sim 0.5 \mathrm{~km} \mathrm{~s}^{-1}\right)$.

\subsection{The Dynamical State of the Turbulent Simulation}

We have repeated the dendrogram analysis for the turbulent simulation using the same algorithm parameters to establish local maxima and contour the data. We adopt a ${ }^{13} \mathrm{CO}-$ to- $\mathrm{H}_{2}$ 


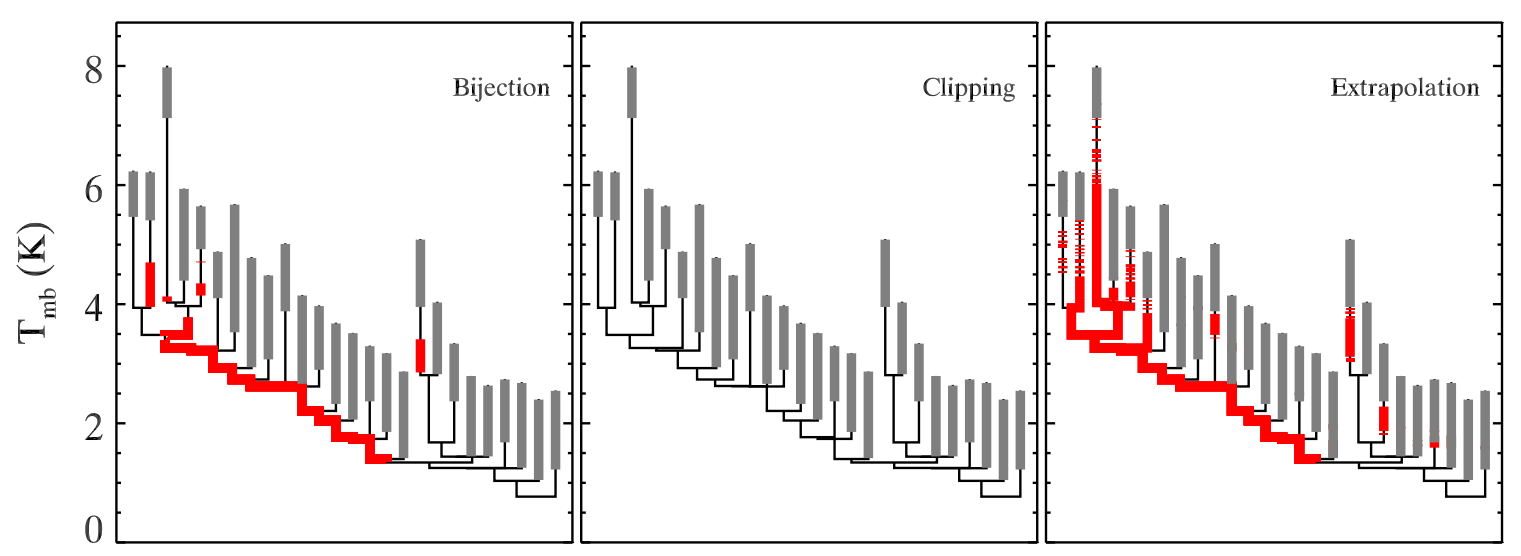

FIG. 11.- Self-gravitating objects in the L1448 dendrograms based on the three different property calculation paradigms presented in $\$ 4.1$ For each dendrogram, regions with $\alpha \leq 2$ are shaded in red and regions where the data quality prohibit calculation of the properties are highlighted in gray. The extent of the self-gravitating regime in parameter space depends on the paradigm adapted though many qualitative features are shared between the bijection and clipping paradigms.

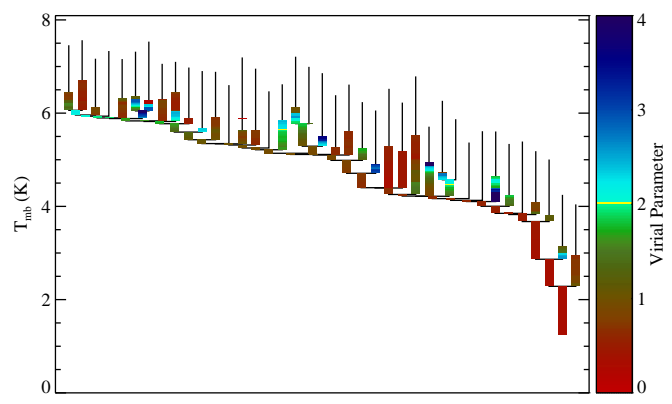

FIG. 12.- Dendrogram of the simulation data cube colored according to the virial parameter at each point. Virial parameter data are suppressed where the errors are larger than $50 \%$. Nearly all of the structure in the simulated data cube corresponds to self-gravitating objects with a few leaves of the dendrogram representing unbound objects.

conversion factor of $X_{2}=10.9$ based on analysis of the simulated ${ }^{13} \mathrm{CO}$ data with respect to the simulated column density, using the same analysis as was used in in $\$ 5.3$. The dendrogram presented in Figure 12, which can be compared to the observed data in Figure 9. The simulated and observed data cubes have similar numbers of leaves (local maxima) in their respective data volumes ( 39 in the simulation vs. 26 in the observations). The span of antenna temperatures are similar, though most of the mergers in the simulated data cubes occur at higher levels than in the observed data. The principal difference between the two dendrograms is that far more of the simulated data cube corresponds to self-gravitating objects than do the actual observations. Regardless of the applicability of the dendrogram interpretations, the analysis illustrates a stark difference in the data cubes. The difference in dynamical states arises from amount of mass in the two data cube. Scaling the total emission in each data cube by the respective conversion factors shows there is $\sim 4$ times as much molecular mass in the simulation cube as there is in the L1448 region, but this extra mass is spread over a similar line width and spatial extent. As a result, self-gravity would play a stronger role in the simulated data cube. The simulation does not include the effects of gravity although our basic analysis suggests that self-gravity would have a significant influence on the simulated region.

\subsection{Interpretation of Dendrogram Properties}

The previous section discusses the physical meaning of the dendrograms under the assumption that the "bijection" paradigm holds relating objects in observational and physical spaces. Previously ( $\$ 4.1$, we presented two other possibilities for relating the observed and physical domains, namely the clipping and extrapolation paradigms. We repeat the calculation of the virial parameter in L1448 for these two possibilities and present the results alongside the bijection results in Figure 11. The extremely conservative clipping paradigm finds no self-gravitating structure in the entirety of the L1448 cloud. Given that simple calculations suggest that the region has a virial parameter of $\alpha \sim 2$ and the presence of star forming clumps at the smallest scales, we conclude that the clipping paradigm is overly conservative and the small structures have more mass than are accorded to them. The extrapolation paradigm finds more self-gravitating structure in the map than the bijection, which is expected since the extrapolation corrects the luminosity by a larger factor than the radius and the line width (see Figure 5). However, it is interesting to note that the same qualitative behavior is present in the extrapolated results as are seen in the bijection analysis. In particular, the analysis finds two dynamically distinct regions in L1448 corresponding to the left- and right-hand branches of the dendrogram. However, the extrapolation results assume that every object should have a brightness profile that runs continuously from the peak value to the zero brightness isosurface, and it may not be applicable in this case. For simplicity, we utilize the bijection scheme for calculating the properties of object substructure although extrapolation may be appropriate in cases where there should be no background emission (see \$6).

\subsection{The Scale of Self-Gravity}

Many previous authors have identified self-gravitating substructure in their analysis of molecular emission. What, then, makes the dendrogram analysis novel? Using by-eye identification (Blitz \& Stark 1986; Bertoldi \& McKee 1992) or automated algorithms such as CLUMPFIND or GAUSSCLUMP invariably finds that the most massive objects on the smallest physical scales sampled by the observations are closest to being self-gravitating (Figure 3 in Bertoldi \& McKee 1992). However, segmentation tends to identify structures on small 


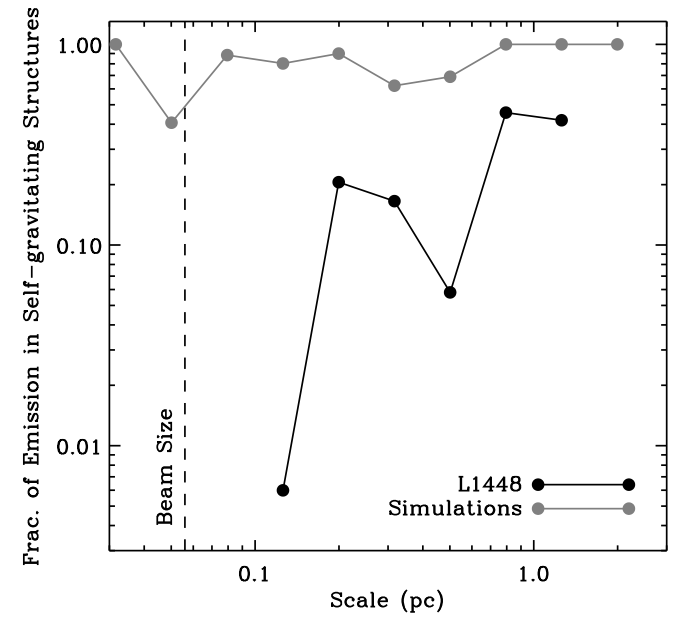

FIG. 13.- Fraction of emission contained within isosurfaces corresponding to self-gravitating objects as a function of size scale for the L1448 ${ }^{13} \mathrm{CO}(1 \rightarrow 0)$ data and the simulated ${ }^{13} \mathrm{CO}$ observations. At small scales, very few objects are self-gravitating, but this fraction grows for larger size scales. The simulations have roughly constant fractions of self-gravitation across size scales.

scales (a few resolution elements), ignoring the objects at larger scales which comprise the superstructure of the molecular cloud. Dendrogram analysis avoids segmentation and naturally includes these larger scales since lower valued isosurfaces encompass more emission with larger spatial extents.

The multi-scale nature of the analysis naturally admits a study such as that shown in the Figure 13 which displays the fraction of emission on given scales in L1448 and in the turbulent simulation that has $\alpha<2$. We construct the diagram by measuring the virial parameter as a function of size scale for all the isosurfaces in the data cube. This fraction is defined as the sum over all isosurfaces

$$
f(R) \equiv \frac{\sum_{i}\left\{L_{i} \mid R_{i} \in[R, R+\Delta R], \alpha_{i} \leq 2\right\}}{\sum_{i}\left\{L_{i} \mid R_{i} \in[R, R+\Delta R]\right\}}
$$

where $L_{i}, R_{i}$ and $\alpha_{i}$ are the luminosity, radius, and virial parameter of the $i$ th isosurface. We bin the virial parameter data into bins of $\Delta R=0.2$ dex in size scale and calculate the fraction of luminosity in each bin contained within isosurfaces corresponding to self-gravitating objects. This calculation illustrates that only a small fraction of the structure at small scales in L1448 corresponds to self-gravitating objects and that fraction grows at larger scales. The saturation of the ${ }^{13} \mathrm{CO}$ line in small, bright regions makes this measurement a lower limit since more of the leaves may correspond to self-gravitating objects than are recovered in this analysis. In contrast, self-gravity is important for nearly all structure at all scales in the simulated observations. Several factors may contribute to this discrepancy. Incomplete physics in the simulation (lack of self-gravity, too high of density) or the incomplete synthesis of spectral line maps (no depletion assumed) may give discrepant results. Alternatively, the comparison may be flawed and the section of the simulation box used may be not be appropriate for comparison to L1448.

\subsection{The Size-Line Width Relationship in L1448}

In addition to identifying sets of isosurfaces that correspond to self-gravitating objects, the dendrogram technique also provides another way to probe the size-line width relationship on intermediate scales inside the molecular clouds.

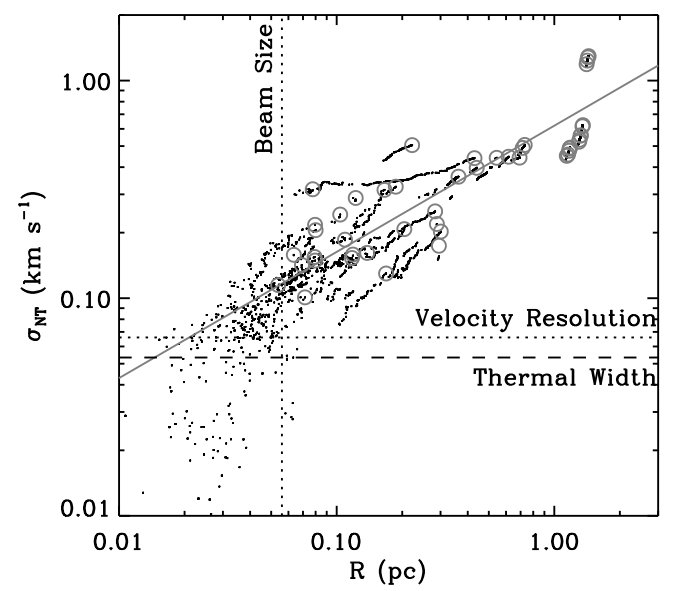

FIG. 14. - The size-line width relationship for the isosurfaces of ${ }^{13} \mathrm{CO}$ emission in L1448. The thermal line width (for $T=10 \mathrm{~K}$ ) and the limits of the instrumental resolution are also indicated in the plot and data below these limits are unreliable. Individual points correspond to isosurfaces used in our contouring. Gray circles indicate the characteristic size and line width for each independent branch in the dendrogram and represent a useful minimum sampling of the data. The gray line indicates the fit to the gray, circled data: $\sigma_{v}=0.6 R^{0.58}$ which is comparable to the size-line width relationship found among turbulent molecular clouds.

The Principal Component Analysis methods developed in Heyer \& Schloerb (1997) and Heyer \& Brunt (2004) are developed to measure the structure function of turbulence within molecular gas and provide an excellent descriptor of the turbulence. The dendrogram application provides a similar measurement by measuring the spatial and velocity extent of the isosurfaces within an emission data cube. Indeed, the virial analysis above can be thought of comparing the spatial and velocity extents of the isosurfaces to the amount of emission they contain. We can construct the size-line width relationship within a data cube by plotting the size vs. the line width of the isosurfaces in the data. This becomes, in effect, a "Type 4" size-line width relationships discussed in Goodman et al. (1998); that is to say, using a single tracer species to measure the relationship in a single cloud.

In Figure 14, we plot the size-line width relationship for the ${ }^{13} \mathrm{CO}$ emission from L1448. A fit to the grey, circled data gives $\sigma_{\mathrm{NT}}=(0.62 \pm 0.04) R_{\mathrm{pc}}^{0.58 \pm 0.04} \mathrm{~km} \mathrm{~s}^{-1}$ typical of the turbulent gas in molecular clouds (Goodman et al. 1998). The scaling relations for turbulent gas traces the data at large scales/line width well. The size has been corrected for beam convolution effects by subtracting the beam width in quadrature. Similarly, the line width has had the thermal contribution of $10 \mathrm{~K}$ gas removed. These corrections are rough since a simple Gaussian deconvolution is inappropriate for the small isosurfaces defined by high brightness contours and the broadening effect of the spectrometer has been neglected. Errors arising from our approximate treatment will become relatively small for sizes and line widths that are significantly larger than the instrumental resolution. At large line widths, linear sets of points correspond to branches in the dendrogram. Along branches, the properties change slowly (see, for example, the radius and luminosity in Figure 5). The discontinuities occur when two isosurfaces merge together resulting in an abrupt change in the size and the line width of the isosurface. At small scales, the data dissolving into a sea of noise since the properties of these surfaces are poorly defined in the face of 
thermal noise and instrumental convolution effects. The scatter around the average relationship results, in part, from the details of the isosurface shapes which vary due to turbulent velocity/density fluctuations but also due to noise.

Dendrograms can also be used to abstract the data set to a defining set of isosurfaces. The gray circles shown in Figure 14 plot a single, characteristic size and line width for each branch of the dendrogram shown in Figure 9 . Since the dendrogram contains 26 leaves, there are 51 independent branches $(2 N-1)$ because each branch is required to join with others in a binary merger. Hence, only one point is plotted for every significantly distinct set of isosurfaces in the data and multiple points from redundant isosurfaces are suppressed.

\section{IDENTIFYING GIANT MOLECULAR CLOUDS}

An additional application of the dendrogram technique is to identify Giant Molecular Clouds (GMCs) in a blended data set. Massive, isolated molecular clouds show virial parameters close to unity (Solomon et al. 1987; Heyer et al. 2001). We propose that GMCs can be identified as the largest-scale self-gravitating structures in the ISM and such structures can be identified in the dendrogram analysis. Unlike the substructure analysis presented previously, we are only interested in the dynamical state of the largest scale emission and the contamination by background emission is likely minimal. Hence, the property calculations for the dendrogram can use the extrapolation paradigm since we are interested in the properties at the $0 \mathrm{~K} \mathrm{~km} \mathrm{~s}^{-1}$ isosurface.

To demonstrate this application of the dendrogram technique, we use the Orion-Monoceros ${ }^{12} \mathrm{CO}$ data of Wilson et al. (2005) taken with the CfA 1.2-m telescope. All of the Orion-Monoceros complex is contained within a single isosurface with $T_{m b}=0.4 \mathrm{~K}$ which must be decomposed into the constituent GMCs. We adopt the standard CO-to- $\mathrm{H}_{2}$ conversion factor $\left(X_{2}=1\right)$ and use the extrapolation paradigm to calculate the virial parameter for each branch of the dendrogram. We adopt a distance to the main Orion complex of $450 \mathrm{pc}$ and use a distance of $800 \mathrm{pc}$ for Monoceros, $425 \mathrm{pc}$ for NGC 2149, and 400 pc for the Northern Filament based on the identifications and distance estimates of Wilson et al. (2005).

Figure 15 shows the dendrogram for the region with sets of isosurfaces that have $\alpha<2$ highlighted. We identify GMCs in an automatic fashion as all emission contained within distinct, self-gravitating regions with masses $M>5 \times 10^{4} M_{\odot}$. The three GMCs in the data cube naturally segregate from the rest of the emission. In Figure 16 we show the emission contained in the $T_{m b}=0.4 \mathrm{~K}$ contour and its characteristic designations. The dendrogram analysis identifies three regions as GMCs and finds that the remaining emission is not sufficiently massive or self-gravitating to be identified as a GMC. Given the good agreement with the standard identifications, we conclude that the dendrogram method can be used to identify GMCs in blended sets of emission. The primary restriction is good knowledge of the distances to different regions of the data volume. This limitation implies that the method is best applied in the outer galaxy or in extragalactic analysis where distances are well-determined.

\section{SUMMARY}

We have presented a new application of tree diagrams to three-dimensional data sets. This application is closely related to the structure tree analysis of Houlahan \& Scalo (1992).
These techniques use dendrograms to represent the merging/bifurcating of contours in a three-dimensional data set as a function of contour level. Each point in the dendrogram corresponds to an isosurface in the data cube. By characterizing molecular emission associated with these isosurfaces, we are able to measure the properties of both small- and large-scale structures in a data set. In particular, we emphasize determinations of the virial parameter and the size-line width relationship at multiple scales in the data. The virial parameter, in particular, provides a means to estimate the influence of selfgravity on a variety of scales in the molecular cloud yielding, for the first time, a uniform study of energetics on a range of scales.

The dendrogram technique is philosophically different from segmentation algorithms such as CLUMPFIND. By preserving and characterizing the hierarchy of emission isosurfaces in a data cube, it is possible to study structures over a range of scales. In principle, the results of the hierarchical decomposition is independent of algorithm parameters, though the actual output is governed by the degree of simplification desired by the user. Although the dendrogram analysis does not segment the data by itself, the results can be used to provide a physically-motivated segmentation of objects in some systems.

Despite the power of the dendrogram technique there are worrisome ambiguities at relating the observed to the physical domain. We have presented three attempts to account for ambiguities in this relationship, but find no satisfactory, universally-applicable method. We note that many of our results are subject to caveats regarding interpretations, but we argue that these caveats do not undermine the applicability of the techniques.

We have analyzed ${ }^{13} \mathrm{CO}(1 \rightarrow 0)$ emission from the L1448 region as observed by the COMPLETE survey of Perseus (Ridge et al. 2006) using the dendrogram methods. We note that common structure analysis techniques have a fundamental scale built into their analysis and tend to analyze the dynamical state of objects on that scale. As such, the synthesis of many such analytic studies conducted on various scales leaves an impression that the dendrogram analysis actually demonstrates. We find self-gravitating structures on all scales in L1448, though not in all regions. In particular, the majority of emission in small-scale structures is not self-gravitating; but, at larger scales, much of the L1448 region is influenced by self gravity.

We have also illustrated the capacity for the dendrogram technique to make differential measurements between data sets. The dendrogram of L1448 is compared to a dendrogram of a theoretical simulation finding qualitative and quantitative differences. Differences of this magnitude were not discernible through other statistical techniques such as determinations of the power spectra (Padoan et al. 2006). Future work will investigate further applications of the differential measurement techniques between dendrograms.

The dendrogram technique can be used to measure the size-line width relationship within molecular clouds using the characteristic sizes and line widths of the constituent isosurfaces in the data. As expected, we recover the typical sizeline width relationship for molecular clouds $\sigma_{v} \propto R^{0.58}$ within a single cloud.

Finally, we conclude the paper by presenting an alternative application of dendrograms: the identification of Giant Molecular Clouds in blended line data sets. We define GMCs 


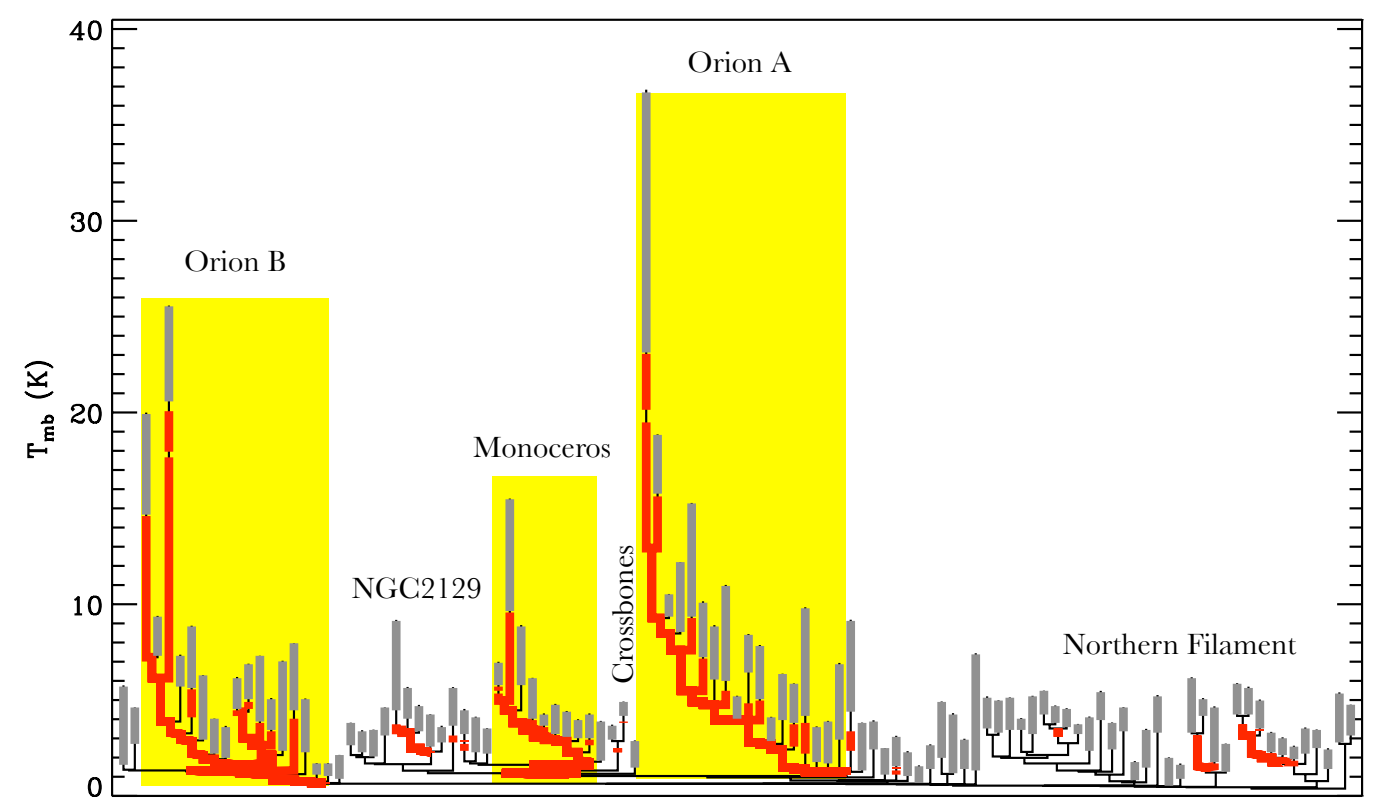

FIG. 15.- The dendrogram of the Orion-Monoceros region. Branches of the dendrogram corresponding to self-gravitating structures are highlighted in red. Regions where the quality of the data prohibit accurate estimation of the virial parameter are shown in gray. The GMCs within the data cube are identified as the largest scale objects that are self-gravitating but not bound to each other. Regions of the dendrogram corresponding to specific objects are labeled and the sections of the dendrogram corresponding to GMCs are shaded in yellow.

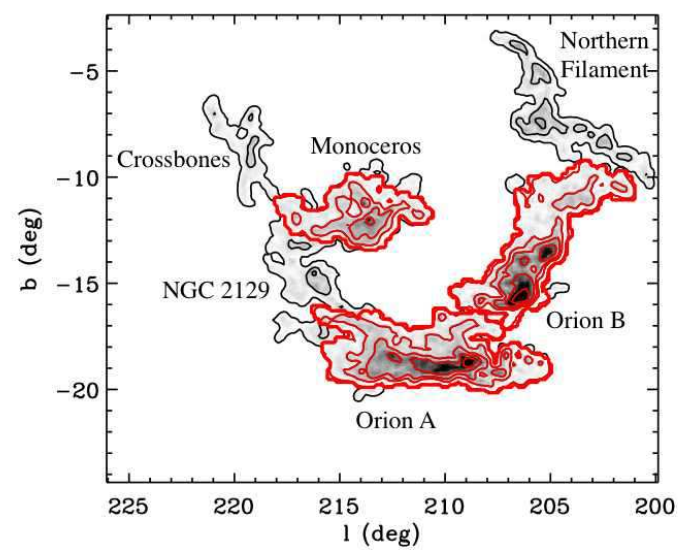

FIG. 16.- Map of emission for the Orion-Monoceros region contained within a $T_{m b}=0.4 \mathrm{~K}$ contour. The three constituent GMCs in the complex have been identified using the dendrogram analysis and their boundaries are indicated in red. The regions are labeled according to their designations in Wilson et al. (2005).

as massive $\left(M>5 \times 10^{4}\right)$ clouds of gas that are (a) selfgravitating but (b) not bound to their surrounding medium. This definition not only identifies GMCs but does so exclusive from including low-mass chaff that is dynamically unrelated to the GMCs. Using this simple definition the dendrogram technique readily identifies the three constituent GMCs in the blended Orion-Monoceros data of Wilson et al. (2005).
Beginning with common application of techniques developed previously, this new perspective on dendrograms illustrates their utility at the visualization and reduction of molecular line data. Dendrograms reduce three dimensional hierarchical data sets to a two dimensional plot that retains essential features regarding the topology of the emission. This reduction is conducted in a fashion that is minimally model dependent, relying on the intrinsic structure of the isosurfaces in an emission line data set.

We are grateful for useful discussions with many people concerning the application, development, and visualization of dendrograms. In particular, we thank Mike Halle, Michelle Borkin, Jonathan Foster, Jonathan Williams, Paola Caselli, and Mark Heyer for constructive comments regarding the development of dendrograms. The comments of an anonymous referee improved the presentation and accuracy of this work. We thank Tom Dame for the use of the Orion-Monoceros data cube. ER's work is funded by an NSF Astronomy and Astrophysics Postdoctoral Fellowship (AST-0502605). JEP is supported by the National Science Foundation through grant \#AF002 from the Association of Universities for Research in Astronomy, Inc., under NSF cooperative agreement AST9613615 and by Fundación Andes under project No. C13442. This material is based upon work supported by the National Science Foundation under Grant No. AST-0407172. This work made extensive use of the NASA's Astrophysics Data System.

\section{REFERENCES}

Alves, J., Lombardi, M., \& Lada, C. J. 2007, A\&A, 462, L17

Ballesteros-Paredes, J., Vázquez-Semadeni, E., \& Scalo, J. 1999, ApJ, 515, 286

Bensch, F. 2006, A\&A, 448, 1043

Bensch, F., Stutzki, J., \& Ossenkopf, V. 2001, A\&A, 366, 636
Bertoldi, F. \& McKee, C. F. 1992, ApJ, 395, 140

Blitz, L., Fukui, Y., Kawamura, A., Leroy, A., Mizuno, N., \& Rosolowsky, E. 2007, in Protostars and Planets V, ed. B. Reipurth, D. Jewitt, \& K. Keil, 81-96

Blitz, L. \& Stark, A. A. 1986, ApJ, 300, L89 
Bohlin, R. C., Savage, B. D., \& Drake, J. F. 1978, ApJ, 224, 132

Brunt, C. M., Kerton, C. R., \& Pomerleau, C. 2003, ApJS, 144, 47

Cernis, K. 1993, Baltic Astronomy, 2, 214

Dame, T. M., Hartmann, D., \& Thaddeus, P. 2001, ApJ, 547, 792

di Francesco, J., Evans, II, N. J., Caselli, P., Myers, P. C., Shirley, Y., Aikawa, Y., \& Tafalla, M. 2007, in Protostars and Planets V, ed.

B. Reipurth, D. Jewitt, \& K. Keil, 17-32

Elmegreen, B. G. 2007, ApJ, 668, 1064

Elmegreen, B. G. \& Falgarone, E. 1996, ApJ, 471, 816

Enoch, M. L., Young, K. E., Glenn, J., Evans, N. J., Golwala, S., Sargent, A. I., Harvey, P., Aguirre, J., Goldin, A., Haig, D., Huard, T. L., Lange, A., Laurent, G., Maloney, P., Mauskopf, P., Rossinot, P., \& Sayers, J. 2006, ApJ, 638, 293

Foster, J. B. \& Goodman, A. A. 2006, ApJ, 636, L105

Ghazzali, N., Joncas, G., \& Jean, S. 1999, ApJ, 511, 242

Goodman, A. A., Barranco, J. A., Wilner, D. J., \& Heyer, M. H. 1998, ApJ, 504,223

Hartmann, L., Ballesteros-Paredes, J., \& Bergin, E. A. 2001, ApJ, 562, 852

Heyer, M. H. \& Brunt, C. M. 2004, ApJ, 615, L45

Heyer, M. H., Carpenter, J. M., \& Snell, R. L. 2001, ApJ, 551, 852

Heyer, M. H. \& Schloerb, F. P. 1997, ApJ, 475, 173

Houlahan, P. \& Scalo, J. 1990, ApJS, 72, 133

-. 1992, ApJ, 393, 172

Kramer, C., Stutzki, J., Rohrig, R., \& Corneliussen, U. 1998, A\&A, 329, 249

Lada, C. J., Muench, A. A., Rathborne, J. M., Alves, J. F., \& Lombardi, M. 2007, ArXiv e-prints, 709

Lada, E. A. 1992, ApJ, 393, L25

Lazarian, A. \& Pogosyan, D. 2000, ApJ, 537, 720

Li, D. \& Goldsmith, P. F. 2003, ApJ, 585, 823

Lombardi, M. \& Alves, J. 2001, A\&A, 377, 1023

McKee, C. F. \& Zweibel, E. G. 1992, ApJ, 399, 551

Motte, F., Andre, P., \& Neri, R. 1998, A\&A, 336, 150

Norman, M. L. \& Bryan, G. L. 1999, in Astrophysics and Space Science Library, Vol. 240, Numerical Astrophysics, ed. S. M. Miyama,

K. Tomisaka, \& T. Hanawa, 19-+

Ostriker, E. C., Stone, J. M., \& Gammie, C. F. 2001, ApJ, 546, 980
Padoan, P., Juvela, M., Kritsuk, A., \& Norman, M. L. 2006, ApJ, 653, L125

Pineda, J. E., Caselli, P., \& Goodman, A. A. 2008, ApJ, accepted (arXiv:0802:0708)

Pineda, J. E., et al.ApJin preparation.

Pound, M. W. \& Goodman, A. A. 1997, ApJ, 482, 334

Press, W. H., Teukolsky, S. A., Vetterling, W. T., \& Flannery, B. P. 1992, Numerical recipes in $\mathrm{C}$. The art of scientific computing (Cambridge: University Press, |c1992, 2nd ed.)

Ridge, N. A., Di Francesco, J., Kirk, H., Li, D., Goodman, A. A., Alves, J. F., Arce, H. G., Borkin, M. A., Caselli, P., Foster, J. B., Heyer, M. H., Johnstone, D., Kosslyn, D. A., Lombardi, M., Pineda, J. E., Schnee, S. L., \& Tafalla, M. 2006, AJ, 131, 2921

Rosolowsky, E. \& Blitz, L. 2005, ApJ, 623, 826

Rosolowsky, E. \& Leroy, A. 2006, PASP, 118, 590

Rosolowsky, E. W., Goodman, A. A., Wilner, D. J., \& Williams, J. P. 1999, ApJ, 524, 887

Scoville, N. Z., Yun, M. S., Sanders, D. B., Clemens, D. P., \& Waller, W. H. 1987, ApJS, 63, 821

Solomon, P. M., Rivolo, A. R., Barrett, J., \& Yahil, A. 1987, ApJ, 319, 730

Strong, A. W. \& Mattox, J. R. 1996, A\&A, 308, L21

Stutzki, J., Bensch, F., Heithausen, A., Ossenkopf, V., \& Zielinsky, M. 1998, A\&A, 336, 697

Stutzki, J. \& Güsten, R. 1990, ApJ, 356, 513

Tafalla, M., Myers, P. C., Caselli, P., \& Walmsley, C. M. 2004, A\&A, 416, 191

Testi, L. \& Sargent, A. I. 1998, ApJ, 508, L91

Ward-Thompson, D., André, P., Crutcher, R., Johnstone, D., Onishi, T., \& Wilson, C. 2007, in Protostars and Planets V, ed. B. Reipurth, D. Jewitt, \& K. Keil, 33-46

West, D. B. 2000, Introduction to Graph Theory (Prentice-Hall)

Williams, J. P., de Geus, E. J., \& Blitz, L. 1994, ApJ, 428, 693

Wilson, B. A., Dame, T. M., Masheder, M. R. W., \& Thaddeus, P. 2005 , A\&A, 430, 523 